\title{
Classification of Microcalcification Clusters via PSO-KNN Heuristic Parameter Selection and GLCM Features
}

\author{
Imad Zyout, PhD \\ Tafila Technical University \\ Tafila, Jordan, 66110
}

\author{
Ikhlas Abdel-Qader, PhD,PE \\ Western Michigan University, Kalamazoo, \\ MI, 49008, USA
}

\begin{abstract}
Texture-based computer-aided diagnosis (CADx) of microcalcification clusters is more robust than the state-of-art shape-based CADx because the performance of shape-based approach heavily depends on the effectiveness of microcalcification (MC) segmentation. This paper presents a texture-based CADx that consists of two stages. The first one characterizes MC clusters using texture features from gray-level co-occurrence matrix (GLCM). In the second stage, an embedded feature selection based on particle swarm optimization and a knearest neighbor $(\mathrm{KNN})$ classifier, called PSO-KNN, is applied to simultaneously determine the most discriminative GLCM features and to find the best $\mathrm{k}$ value for a KNN classifier. Testing the proposed CADx using $25 \mathrm{MC}$ clusters from mini-MIAS dataset produced classification accuracy of $88 \%$ that obtained using 2 GLCM features.
\end{abstract}

\section{Keywords}

Microcalcifications, GLCM texture features, feature selection, particle swarm optimization

\section{INTRODUCTION}

Computer-aided diagnosis (CADx) is mainly intended to provide radiologists with a second opinion that might improve the positive predictive value of mammography. The development of robust feature extraction and automated feature selection methods are the major demands of the current computer-aided diagnosis technology in mammography [1],[2]. Unlike the state of the art shape-based CADx of mammographic MC clusters, a texturebased CADx does not need a microcalcification segmentation stage. Another advantage of texture based schemes is their ability to characterize texture dependency and spectral properties, which cannot be characterized using shape measures.

Commonly used texture features are those derived by using Laws measure of texture, spectral descriptors, and texture features derived from gray-level co-occurrence matrices (GLCMs) [2]. Among these approaches, GLCM based texture analysis is very common and has been examined in several studies [3]-[9]. Dhawan et al. [3] used a heuristic feature search based on GA and neural network classifier to classify 191 MC clusters from a local dataset. Using shape and GLCM texture features and a GA-based feature selection to diagnose $145 \mathrm{MC}$ clusters, Chan et al. [4] achieved an area under the ROC curve of 0.89. An earlier study [5] by Zadeh and his colleagues demonstrated the superior performance of the shape features over GLCM features. In a more detailed study and using same mammographic dataset, Zadeh et al.[6] achieved an area under the receiver operating characteristic (ROC) curve of 0.89 using texture features derived using multi-wavelet transforms, feature selection using GAs, and a KNN classifier. The results of this study demonstrate that texture features from multi-wavelets are more superior than other features including wavelet packets, GLCM, and shape features. Zadeh et al. [6] also demonstrated that including the background texture when extracting GLCM features of MCs produced better results than only characterizing the texture of the regions representing individual MCs. Singh et. al [7] combined shape, spectral, and GLCM features to classify microcalcifications using kernelbased support vector machine classifiers. Hamdi et al. [8] classified MC clusters from MIAS database using GLCM and spectral features, feature selection using Fisher discriminate analysis (FDA), and a KNN classifier. Other studies [9], [10] demonstrated that it is not the texture of MCs objects but it the texture of breast tissue surrounding microcalcifications that can be useful for cancer diagnosis.

Most texture-based CADx systems [3]-[7] used a heuristic feature search based on GA approach and a KNN classifier for accomplishing the feature selection and pattern classification stages, respectively. Also, none of the previous studies has incorporated the feature selection during the classifier learning and parameter selection stages. Hence, this paper presents a PSO-KNN embedded feature selection framework for solving the model selection problem associated with texture-based diagnosis of microcalcification clusters. This PSO-KNN uses a heuristic parameter search based on a particle swarm optimization (PSO) algorithm for selecting the best GLCM features and for optimizing the performance of the KNN classifier. Advantages of performing parameter selection using an embedded PSO-KNN scheme include utilizing a PSO heuristic parameter search that is computationally more attractive than GA approaches [11] and integrating the feature selection and classifier's parameter optimization tasks that is more efficient than performing each task individually. 
The organization of the remaining sections of this paper is as follows: the theoretical background of the GLCM texture features and heuristic search using PSO are briefly explained in Section 2. Section 3 describes the proposed computed aided diagnosis using texture features and PSO-KNN feature selection and classification approach. The experimental results and conclusions are discussed in Sections 4 and 5, respectively.

\section{BACKGROUND \\ 2.1 Texture analysis using GLCM features}

Analysis of image texture using a gray level co-occurrence matrix (GLCM), that is also known as spatial gray-level dependence matrix (SGLDM), was first applied for feature extraction and diagnosis Alzheimer's disease [12]. During the last two decades, GLCM based texture analysis has become a very popular feature extraction method for the detection and classification masses and microcalcifications in digital mammography [1], [3]-[9].

A gray-level co-occurrence matrix analyzes second order statistics of the gray-level histogram of an image. This step is done by measuring the probability of the occurrence of different gray-level patterns. Such patterns are formed by two gray-levels spatially separated by a distance $d$ and located along a line oriented at angle $\theta$. These orientations are represented by the four neighboring pixels of the pixel located at $\mathrm{x}$ and $\mathrm{y}$. Orientation angle $\theta$ takes four values: $0^{\circ}$ (horizontal), $45^{\circ}$ (diagonal), $90^{\circ}$ (vertical), and $135^{\circ}$ (anti-diagonal). These angles represent the orientation of four neighboring pixels of a pixel (x,y). For an image $I$ of size $M \times N$ and L gray-levels, a GLCM of size $L \times L$ is computed as

$\mathrm{p}(i, j, d, \theta)=\frac{1}{N_{d, \theta}} \sum_{x=1}^{M} \sum_{y=1}^{N} P\left\{I(x, y)=i, I\left(x \pm d . u_{\theta}, y \pm d . v_{\theta}\right)=j\right\}$

where $p\{$.$\} is 1$ if the condition described by the argument is satisfied. $(i, j)$ are gray-level values, orientation indicator is given by $\left(v_{\theta}, u_{\theta}\right)$, which is $(1,0)$ for $\theta$ equals $0^{\circ},(0,1)$ for $90^{\circ}$, and $(1,-1)$ for $45^{\circ}$, and $(-1,1) 135^{\circ}$. Moreover, $N_{d, \theta}$ is a normalizing factor used to make the sum of all GLCM entries equal to 1 .

\subsection{Particle swarm optimization}

Exhaustive parameter and feature selection guarantee a global solution to the problem of model selection in machine learning. However, the major shortcomings of this method are the computationally complexity of the process and the poor generalization of the solution. This makes heuristic optimization methods such as PSO and GAs more appropriate for solving the model selection task.
The idea of the heuristic optimization using a particle swarm optimization method [13] is similar in principle to other population based methods such as Genetic and evolutionary algorithms [14]. The heuristic parameter selection based on a PSO algorithm is started by randomly initializing the candidates' solution (i.e. members of the swarm) in the parameter space. For this study, the parameter space consists of the feature space and the classifier's parameter space. Then, PSO particles move and collaborate to find the optimal solution to the parameter selection task.

\subsection{K-nearest neighbor (KNN) classifier}

A KNN classifier is one of the most straightforward supervised learning machines that continues to perform very well in solving many pattern recognition problems including diagnosis of MC clusters [2]. Basically, pattern classification using a KNN classifier uses experience from the previous training patterns. That is an input test data is classified into a certain class, a malignant or benign in this study, by using majority voting among the nearest $\mathrm{k}$ neighbors. This $\mathrm{k}$ nearest neighbors are selected by using a predefined distance metric (correlation, Euclidian distance) to compute and select the nearest training patterns that are neighbor to input sample in the sense of the selected metric.

\section{TEXTURE-BASED CADX USING PSO- KNN}

The proposed texture-based CADx of MC cluster as shown by Figure 1 consists of two stages. In the first stage, a mammographic region depicting MC cluster is characterized using second order statistics of gray-levels. The second stage represents parameter selection using PSO-KNN framework, which involves reducing the dimensionality of the feature space, selecting the optimal subset of GLCM features, and tuning the classifier parameters to improve the generalization ability of the classification process.

\subsection{GLCM texture features}

From each GLCM matrix, various texture descriptors can be computed. In this paper, we have measured a group of 14 GLCM descriptors that are commonly used to characterize MC clusters in mammography. GLCM descriptors measured in this paper are: energy, correlation, homogeneity, contrast, entropy, sum of squares, sum average, sum entropy, difference average, difference variance, difference entropy, two information measures of correlation, and Inverse difference normalized.

Instead of using GLCM descriptors computed from each directional GLCM matrix, combining the values of each descriptor is usually used to form a summary GLCM feature [6]. One approach for generating a summary feature from the four directional ones is by computing some statistical moments such as average, standard deviation, and range of a given GLCM descriptor [6]. In addition to computing summary features by averaging, we use the range of the four directional GLCM descriptors. This process leads to a set of 28 GLCM features. 


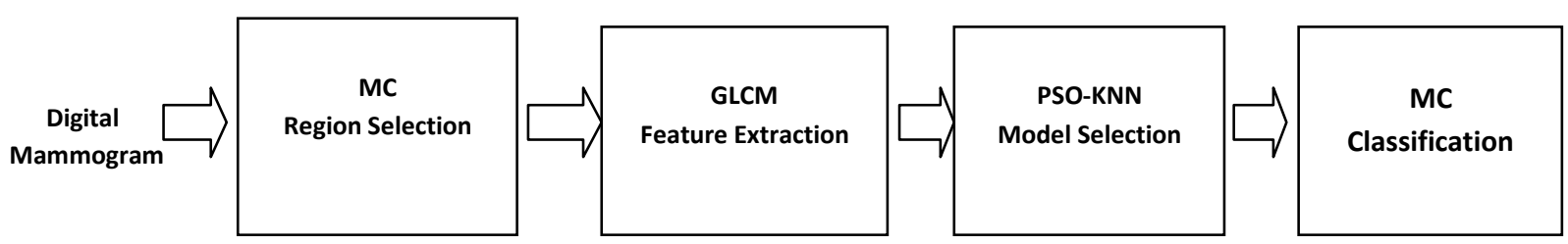

Fig1: Classification of microcalcification clusters using PSO-KNN and GLCM texture features.

\subsection{Parameter selection using PSO-KNN}

Model or parameter selection using a PSO-KNN embedded strategy represents a unified scheme for feature selection and for tuning the parameter of the classifier. The presented PSO-KNN heuristic method uses a binary feature search to select the most discriminative GLCM features.

PSO-KNN also optimizes the performance of a KNN classifier by finding the best $\mathrm{k}$ value that produces the best classification performance. Hence, the PSO-KNN framework uses a discretevalued PSO [15] with particles of 29 coordinates to accomplish the model selection process.

The fitness of each PSO-KNN candidate's solution is evaluated using leave-one-out cross validation method. The objective function of the POS-KNN optimization is defined as the product of true positive fraction (classification sensitivity), true-negative fraction (classification specificity), and penalization factor that decreases as the size of the selected feature subset increases.

\section{EXPERIMENTAL RESULTS}

\subsection{Test data}

The proposed texture based CADx, including the GLCM features and PSO-kNN heuristic model selection, has been investigated using mammographic regions with proven biopsy mammographic microcalcification clusters. These regions were obtained from mini-MIAS dataset [16] that contains 20 mammogram with 25 MC clusters of which 12 are malignant and 13 are benign. Samples of this test data are shown in Figure 2.

\subsection{Implementation and experiment setup}

All feature extraction, heuristic search and parameter selection using a PSO algorithm, and pattern classification using a KNN classifier are implemented in MATLAB software. For the heuristic model selection using PSO-KNN method, the size of the population and the number of iterations were set to 100 and 50, respectively. Moreover, we used typical values from [17] to control the search process of the PSO algorithm.

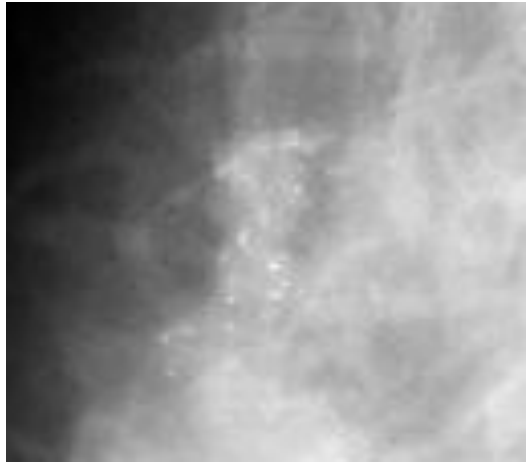

(a) Malignant (mdb241)

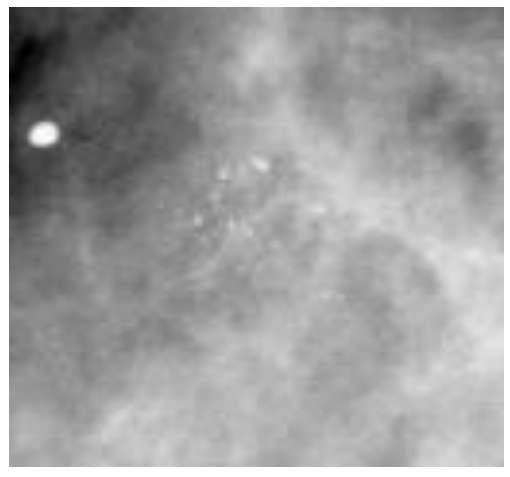

(b) Benign (mdb223)

Fig 2: Examples of microcalcification clusters from mini-MIAS public dataset. 


\subsection{Results Analysis}

The results of the proposed classification and model selection methods are presented in Table 1 and Table 2. In this work, the fitness function of the PSO-KNN estimated using leave-one-out cross validation is designed to find a learning model that produces the highest sensitivity or true positive fraction, highest specificity or true negative fraction, and the smallest size of the feature subset. The main reason for adopting this type of fitness function instead of the classification accuracy alone is that we are trying to find the solution that have lower tendency to over fit the training data.

As presented by Table 1 , the best classification performance produced by PSO-KNN model selection of is of $88 \%$ accuracy that corresponds to sensitivity of $100 \%(0 \mathrm{FN})$ and specificity of $77 \%$ (3 FP). This result is achieved using two GLCM features, which represented by the average of homogeny and the range of the contrast. Another learning model that also produces accuracy of $88 \%$ and demonstrates a trade-off of the specificity and sensitivity rates is achieved by using a set of three GLCM features.

These features include the average of difference entropy, range of difference variance, and range of information of correlation I.

This paper also has examined the impact of the size of mammographic texture used to characterize micro-calcification clusters on the discriminative power of the GLCM features and its affect on the outcomes of feature selection using a PSO-KNN method. Table 2 results of MC clusters classification using GLCM features extracted from three different mammographic regions of size of $128 \times 128,256 \times 256$, and $512 \times 512$ pixels. Because some MC clusters are extracted from the same mammogram, using larger region sizes as $256 \times 256$ and $512 \times$ 512 pixels has led to merging these MC clusters into one region with two or three clusters. For instance, two benign clusters, extracted from mdb223 mammogram, enclosed using two region of size $128 \times 128$, are combined when the region size is $256 \times$ 256 or $512 \times 512$ pixels. This process is illustrated by Figure 3 . This process reduced the size of dataset from 25 in case of $128 \times$ 128 region size to 22 and 21 for $256 \times 256$ and $512 \times 512$ sizes, respectively.

As demonstrated by Table 2, the classification accuracy has not significantly degraded by using larger mammographic regions of size of $256 \times 256$ and $512 \times 512$ pixels. That is using GLCM features extracted from $512 \times 512$ pixels; we achieved classification accuracy of $90 \%$ that corresponds to 1 FP and 1 FN.

Table 1. Results of classifying MC clusters using PSO-KNN feature and parameter selection method. *

\begin{tabular}{|c|c|c|c|c|c|}
\hline $\mathbf{k}$ & $\mathbf{N}^{* *}$ & Sensitivity $\backslash \mathbf{F N}$ & Specificity $\backslash \mathbf{F P}$ & Accuracy & Fitness value \\
\hline 3 & 3 & $0.92 \backslash 1$ & $0.85 \backslash 2$ & 0.88 & 0.28 \\
\hline 3 & 2 & $1.0 \backslash 0$ & $0.77 \backslash 3$ & 0.88 & 0.26 \\
\hline 3 & 1 & $0.83 \backslash 2$ & $0.77 \backslash 3$ & 0.80 & 0.36 \\
\hline 3 & 5 & $0.83 \backslash 2$ & $0.92 \backslash 1$ & 0.88 & 0.33 \\
\hline 3 & 8 & $0.92 \backslash 1$ & $0.85 \backslash 2$ & 0.88 & 0.40 \\
\hline
\end{tabular}

*Size of mammographic region used to derive GLCM features is $128 \times 128$ pixels.

$* * \mathrm{~N}$ is the size of the feature subset.
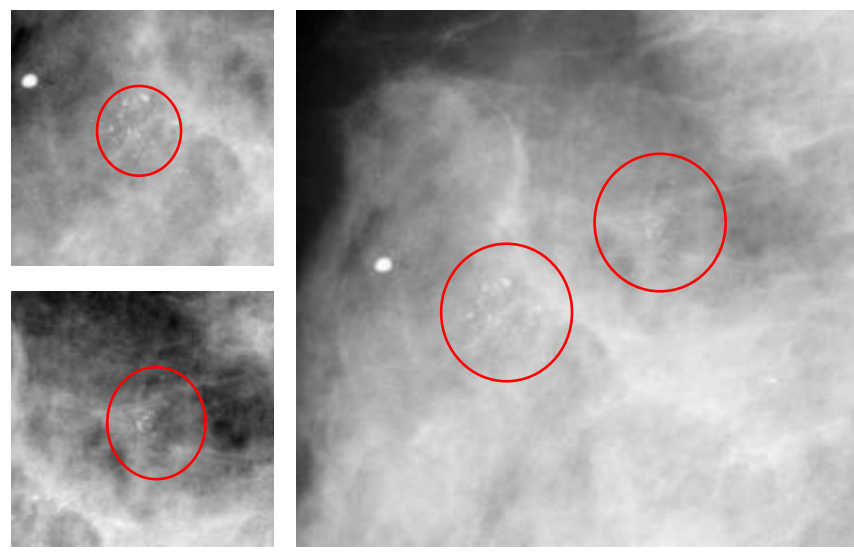

Fig. 3: An example of merging MC clusters enclosed by regions of $128 \times 128$ pixels into a region of $256 \times 256$ pixels. 
This result, which produced using 4 GLCM texture features, might indicate the positive impact of the tissue surrounding MC cluster [9].

Table 2. Impact of the mammographic region size on the classification using GLCM features.

\begin{tabular}{|l|c|c|c|c|}
\hline $\begin{array}{c}\text { Size } \\
\text { ( in pixels) }\end{array}$ & $\mathbf{N}$ & Sensitivity & Specificity & Accuracy \\
\hline $128 \times 128$ & 3 & 0.92 & 0.85 & 0.88 \\
\hline $256 \times 256$ & 9 & 0.82 & 0.91 & 0.86 \\
\hline $512 \times 512$ & 4 & 0.90 & 0.90 & 0.90 \\
\hline
\end{tabular}

Results of this study also demonstrated the impact of the region size on the set of GLCM features with the strongest discriminating power. Although the set of GLCM features are different from one region size to another, some GLCM futures such as the sum average and the sum difference were common among region sizes.

Diagnosis of $\mathrm{MC}$ clusters in mammography has been investigated in several studies [2]-[10], [18],[19]. CADx systems presented in these studies were different in terms of the feature spaces, feature selection methods, and the classifiers used for discriminating malignant and benign clusters, and datasets used for evaluating the proposed schemes. Using different datasets makes any direct comparison impropriate. Hence, the comparison presented in this paper only includes CADx studies used MIAS dataset for performance evaluation. Using shape features and a full model selection based on PSO-SVM approach, Zyout et al. [2] obtained a perfect $(100 \%)$ classification accuracy. Hamdi et al. [8] obtained a classification accuracy of $95.5 \%$ using 6 GLCM features and a KNN classifier. Papadopoulos et al. [18] obtained an area under ROC (Az) of 0.81 and 0.78 using SVM and ANN classifiers, respectively. Further, Wang et al. [19] used mixed texture and shape features and GA for SVM model selection and dimensionality reduction of the feature space and achieved $\mathrm{Az}$ of 0.86 .

\section{CONCLUSIONS}

This paper has classified MC clusters using GLCM features and PSO-KNN embedded feature selection approach. The results of classifying MC clusters from mini-MIAS have revealed the effectiveness of feature selection, dimensionality reduction, and classifier parameter selection using PSO-KNN. Moreover, results of examining the relation between the size mammographic regions used to compute GLCM features and the discriminative power of GLCM features indicated the positive impact of the texture surrounding MC clusters. Extension of the proposed PSO-KNN approach to other feature spaces and validating the results of this paper using a larger dataset of mammograms are planned as an ongoing and future work of this paper.

\section{REFERENCES}

[1] Elter, M. and Horsch, A. 2009. CADx of mammographic mass and clustered microcalcifications: A review. Medical Physics, 36(6), 2052-2068.

[2] Zyout, I. 2010. Toward automated detection and diagnosis of mammographic microcalcifications. Doctoral dissertation, Dept. of Elect. \& Comp. Eng., Western Michigan University.

[3] Dhawan, A. P., Chitre, Y., Bonasso, C., and Wheele, K. 1995. Radial-basis-function-based classification of mammographic microcalcifications using texture features. In Proceedings of the 17th Annual International Conference and 21st Canadian Medical and Biological Engineering Conference, 535-536.

[4] Chan, H. P., Sahiner, B., Lam, K. L., Petrick, N., Helvie, M. A., Goodsitt, M. M., and Adler, D. D. 1998. Computerized analysis of mammographic microcalcifications in morphological and texture feature spaces. Medical Physics, 2007-2019.

[5] Zadeh, H. S., Nezhad, P.S., and Rad, F. R. 2001. Shape based and texture-based feature extraction for classification of microcalcifications in mammograms. In Proceedings of SPIE Medical Imaging, 4322, 3010-310.

[6] Zadeh, H. S., Rad, F. R., and Nejad, S. P. 2004. Comparison of multiwavelet, wavelet, Haralick, and shape features for microcalcification classification in mammograms. Pattern Recognition, 37, 1973-1986.

[7] Singh, S., Kumar, V., Verma, H. K., and Singh, D. 2006. SVM Based System for classification of Microcalcifications in Digital Mammograms. In proceeding of the 28th IEEE EMBS Annual International Conference, New York City, USA.

[8] Hamdi, N., Auhmani, K., and Hassani, M. M. 2008. Design of a high-accuracy classifier based on fisher discriminate analysis: Application to ComputerAided Diagnosis of Microcalcifications. In Proceedings of the International Conference on Computational Sciences and its Applications ( ICCSA 2008).

[9] Karahaliou, A., Boniatis, I., Sakellaropoulos,P., Skiadopoulos, S., Panayiotakis, G., and Costaridou, L. 2007. Can texture of tissue surrounding microcalcifica-tions in mammography be used for breast cancer diagnosis? Nuclear Instruments and Methods in Physics Research, 580, 1071-1074.

[10] Thiele, D. L., Kimme-Smith, C., Johnson, T. D., McCombs, M., and Bassett, L. W. 1996. Using tissue texture surrounding calcification clusters to predict benign vs malignant outcomes. Medical Physics, 23, 549-555.

[11] Guo, X. C. , Yang, J. H., Wu, G. C., Wang, C. Y., and Liang, Y. C. 2008. A novel LS-SVMs hyper- 
parameter selection based on particle swarm optimization. Neurocomputing, 71, 3211-3215.

[12] Haralick, R. M. 1979. Statistical and structural approaches to texture. In Proceedings of IEEE, 67 (5), 786-804.

[13] Kennedy, J. and Eberhart, R. 1995. Particle swarm optimization. In Proceedings of the IEEE International Conference on Neural Networks, Perth: IEEE Service Center, Piscataway, NJ, 4, 1942-1948.

[14] Siedlecki, W. and Sklansky, J. 1989. A note on genetic algorithm for large scale feature selection. Pattern recognition letter, 10, 335-347.

[15] Kennedy, J. and Eberhart, R. C. 1997. A discrete binary version of the particle swarm algorithm. In Proceedings of the Conference on Systems, Man, and Cybernetics, Piscataway, NJ, 4104-4109.
[16] Suckling, J., Parker, J., Dance, D., Astley, S., Hutt, I., Boggis, C., Ricketts, I., Stamatakis, E., Cerneaz, N., Kok, S., Taylor, P., Betal, D., and Savage, J. 1994. The mammographic image analysis society digital mammogram database. Exerpta Medica, 1069, 375-378.

[17] Escalante, H. J., Montes, M., and Sucar, L. E. 2009. Particle Swarm Model Selection. Journal of Machine Learning Research, 10, 405-440.

[18] Papadopoulos, A., Fotiadis, D. I., and Likas, A. 2005. Characterization of clustered microcalcifications in digitized mammograms using neural networks and support vector machines. Artificial Intelligence in Medicine, 4( 2),141-150. 